\title{
CONVEXITY AND MINIMAL GERSCHGORIN SETS ${ }^{1}$
}

B. W. LEVINGER

1. The Gerschgorin circle theorem gives bounds for the eigenvalues of an $n \times n$ complex matrix $A=\left(a_{i, j}\right)$ in terms of the diagonal elements and the moduli of the off-diagonal elements. Thus these bounds apply equally well to any matrix in the class

(1) $\Omega_{A}=\left\{B=\left(b_{i, j}\right)\left|b_{i, i}=a_{i, i},\right| b_{i, j}|=| a_{i, j} \mid, 1 \leqq i, j \leqq n\right\}$.

The problem of how to determine all eigenvalues of matrices in $\Omega_{A}$ was solved by R. S. Varga and the author [6], [4] by the introduction of minimal Gerschgorin sets, $G^{\phi}\left(\Omega_{A}\right)$, to be defined in $\S 3$.

The original proofs depended strongly upon the Perron-Frobenius theory of nonnegative matrices. In this note, we give a new derivation of the main results of [4] using a lemma of V. Klee on convex sets [3].

I am indebted to D. E. Bzowy for the proof of Theorem 2.

2 . In real $n$-dimensional space $R_{n}$ we define the following subsets.

$$
\begin{aligned}
& H=\left\{\mathrm{x} \in R_{n} \mid \sum_{i=1}^{n} x_{i}=1, x_{i} \geqq 0,1 \leqq i \leqq n\right\}, \\
& Q_{j}=\left\{\mathrm{x} \in H \mid x_{j}=0\right\}, \quad 1 \leqq j \leqq n .
\end{aligned}
$$

Given $n^{2}$ constants $c_{i, j} \geqq 0,1 \leqq i, j \leqq n$, let

$$
\begin{aligned}
M_{i, j} & =\left\{\mathrm{x} \in H \mid c_{i, j} x_{j} \leqq \sum_{k \neq j} c_{i, k} x_{k}\right\}, \quad 1 \leqq i, j \leqq n, \\
S_{j} & =\bigcap_{i=1}^{n} M_{i, j}, \quad 1 \leqq j \leqq n .
\end{aligned}
$$

The sets $H, Q_{j}, S_{j}, M_{i, j}$, are compact convex sets in $R_{n}$ and, for all $i, j=1, \cdots, n$,

$$
Q_{j} \subset S_{j} \subset M_{i, j}
$$

We give two theorems relating the sets $S_{j}, M_{i, j}$, and $H$.

ThEOREM 1. Let $\phi$ be any permutation of the integers $1, \cdots, n$. Then the following conditions are equivalent:

$$
\bigcap_{i=1}^{n} M_{i, \phi(i)} \neq \varnothing,
$$

Received by the editors October 23, 1966.

1 This research was supported in part by NSF Grant GP-5553. 


$$
\bigcup_{i=1}^{n} M_{i, \phi(i)}=H
$$

Proof. (7) implies (8): Let $y \in H$ and $z \in \bigcap_{i=1}^{n} M_{i, \phi(i)}$. We may assume that $y \neq z$, and choose $k$ such that $\min _{z_{i} \neq 0} y_{i} / z_{i}=y_{\phi(k)} / z_{\phi(k)}$ $=\lambda<1$. Then $\tilde{\boldsymbol{y}}=(1-\lambda)^{-1}(\boldsymbol{y}-\lambda z)$ is in $H$ and $\tilde{y}_{\phi(k)}=0$. Thus $\tilde{y} \in Q_{\phi(k)}$ $\subset M_{k, \phi(k)}$, and, since $M_{k, \phi(k)}$ is convex, $y=(1-\lambda) \tilde{y}+\lambda z \in M_{k, \phi(k)}$.

(8) implies (7): This is a consequence of the following lemma of V. Klee [3]: If $(j+1)$ closed convex sets in $R_{n}$ have convex union and any $j$ have a common point, then there is a point common to all. By assumption $\bigcup_{i=1}^{n} M_{i, \phi(i)}=H$ which is convex, and from (6) $\bigcap_{i \neq j} M_{i, \phi(i)}$ $\supset \bigcap_{i \neq j} Q_{\phi(i)} \neq \varnothing$, so that any $(n-1)$ sets $M_{i, \phi(i)}$ have nonempty intersection. Thus $\bigcap_{i=1}^{n} M_{i, \phi(i)} \neq \varnothing$.

THEOREM 2. The following three conditions are equivalent:

(9) $H=\bigcup_{j=1}^{n} S_{j}$.

(10) $\bigcap_{1 \leq i, j \leq n} M_{i, j} \neq \varnothing$.

(11) For any permutation $\phi$ of $1, \cdots, n$,

$$
H=\bigcup_{i=1}^{n} M_{i, \phi(i)}
$$

Proof. (9) implies (10): The $n$ closed convex sets $S_{j}$ have convex union $H$. Any $(n-1)$ of the $S_{j}$ have a common point, since by (6), $\varnothing \neq \bigcap_{j \neq i} Q, \subset \bigcap_{j \neq i} S_{j}$. Thus by Klee's lemma, $\varnothing \neq \bigcap_{j=1}^{n} S_{j}$ $=\bigcap_{1 \leqq i, j \leqq n} M_{i, j}$.

(10) implies (11): This follows immediately from Theorem 1.

(11) implies (9): If $H \neq \bigcup_{j=1}^{n} S_{j}$, then $\exists x \in H$ such that $\mathrm{x} \notin S_{j}$, for $j=1, \cdots, n$. Consequently, for each $j=1, \cdots, n$, we can find $\phi(j)$ such that $\mathrm{x} \notin M_{\phi(j), j}$, or by (4) that $c_{\phi(j), j} x_{j}>\sum_{k \neq j} c_{\phi(j), k} x_{k}$. From this, it follows that, for each $j=1, \cdots, n$ and $k \neq j, \mathrm{x} \in M_{\phi(j), k}$ and thus, that if $k \neq j, \phi(k) \neq \phi(j)$. Hence $\phi$ is a permutation and $\mathrm{x} \notin \mathrm{U}_{j=1}^{n} M_{\phi(j), j}$ contradicting (11).

It can be shown that, if $H \neq \bigcup_{j=1}^{n} S_{j}$, the permutation $\phi$ constructed above such that $H \neq \bigcup_{j=1}^{n} M_{\phi(j), j}$ is unique [5, Theorem 4].

Theorems 1 and 2 may be generalized to apply to sets of the form

$$
\bar{M}_{i, j}=\left\{x \in H \mid c_{i, j, j} x_{j} \leqq \sum_{k \neq j} c_{i, j, k} x_{k}\right\}, \quad 1 \leqq i, j \leqq n,
$$

where $c_{i, j, k} \geqq 0,1 \leqq i, j, k \leqq n$, provided

$$
c_{i, j, j} x_{j}>\sum_{l \neq j} c_{i, j, l} x_{l} \quad \text { implies } \quad c_{i, k, k} x_{k} \leqq \sum_{l \neq k} c_{i, k, l} x_{l}
$$


for any $i, j=1, \cdots, n$ and $k \neq j$. The proofs for the general case are identical.

3. We briefly recall some definitions from [4]. For any complex matrix $A$, let $\Omega_{A}$ be given by (1) and

$$
S\left(\Omega_{A}\right)=\left\{z \mid \operatorname{det}(z I-B)=0 \text { for some } B \in \Omega_{A}\right\} .
$$

If $\phi$ is any permutation of $1, \cdots, n$ and $x \in H$, we define

$$
\begin{array}{r}
G_{i}^{\phi}(\mathrm{x})=\left\{\sigma|| a_{i, \phi(i)}-\sigma \delta_{i, \phi(i)}\left|x_{\phi(i)} \leqq \sum_{j \neq \phi(i)}\right| a_{i, j}-\sigma \delta_{i, j} \mid x_{j}\right\}, \\
1 \leqq i \leqq n,
\end{array}
$$

$$
\begin{aligned}
G^{\phi}(\mathrm{x}) & =\bigcup_{i=1}^{n} G_{i}^{\phi}(\mathrm{x}), \\
G^{\phi}\left(\Omega_{A}\right) & =\bigcap_{\mathbf{x} \in H} G^{\phi}(\mathbf{x}) .
\end{aligned}
$$

The main results of [4] were a characterization of $G^{\phi}\left(\Omega_{A}\right)$ and a proof that

$$
S\left(\Omega_{A}\right)=\bigcap_{\phi} G^{\phi}\left(\Omega_{A}\right)
$$

The former is a simple consequence of Theorem 1 and we will derive the latter from Theorem 2.

Theorem 3. $\sigma \in G^{\phi}\left(\Omega_{A}\right)$ ifandonly if $\exists \mathbf{x} \in H$ such that, for $i=1, \cdots, n$,

$$
\left|a_{i, \phi(i)}-\sigma \delta_{i, \phi(i)}\right| x_{\phi(i)} \leqq \sum_{j \neq \phi(i)}\left|a_{i, j}-\sigma \delta_{i, j}\right| x_{j} .
$$

Proof. For any $i, j=1, \cdots, n$, let

$$
M_{i, j}(\sigma)=\left\{x \in H|| a_{i, j}-\sigma \delta_{i, j}\left|x_{j} \leqq \sum_{k \neq j}\right| a_{i, k}-\sigma \delta_{i, k} \mid x_{k}\right\} .
$$

By (14), (15), for any $x \in H, \sigma \in G_{i}^{\phi}(x)$ if and only if $x \in M_{i, \phi(i)}(\sigma)$, and $\sigma \in G^{\phi}(\mathbf{x})$ is equivalent to $\mathrm{x} \in \mathrm{U}_{i=1}^{n} M_{i, \phi(i)}(\sigma)$. Thus, $\sigma \in G^{\phi}\left(\Omega_{A}\right)$ $=\bigcap_{\mathbf{x} \in H} G^{\phi}(\mathbf{x})$ if and only if $\bigcup_{i=1}^{n} M_{i, \phi(i)}(\sigma)=H$, which is equivalent to $\bigcap_{i=1}^{n} M_{i, \phi(i)}(\sigma) \neq \varnothing$, by Theorem 1 . This completes the proof of our theorem, which is equivalent to Theorem 1 of [4].

To prove (17), we first give a characterization of $S\left(\Omega_{A}\right)$.

Lemma. $\sigma \in S\left(\Omega_{A}\right)$ if and only if $\exists x \in H$ such that, for $1 \leqq i, j \leqq n$,

$$
\left|a_{i, j}-\sigma \delta_{i, j}\right| x_{j} \leqq \sum_{k \neq j}\left|a_{i, k}-\sigma \delta_{i, k}\right| x_{k} .
$$


Proof. $\sigma \in S\left(\Omega_{A}\right)$ if and only if $\exists B \in \Omega_{A}$ and $y \neq 0$, such that $(B-\sigma I) \boldsymbol{y}=\mathbf{0}$. Taking components and using the definition (1) of $\Omega_{A}$, we obtain, for $1 \leqq j \leqq n$,

$$
0=\sum_{k=1}^{n}\left(b_{j, k}-\sigma \delta_{j, k}\right) y_{k}=\sum_{k=1}^{n}\left|a_{j, k}-\sigma \delta_{j, k}\right|\left|y_{k}\right| \exp \left(i \theta_{j, k}\right),
$$

where the $\theta_{j, k}$ are real numbers and $\theta_{j, j}=0,1 \leqq j, k \leqq n$. Thus for each $j=1, \cdots, n$, the $n$ complex numbers,

$$
\lambda_{j, m}=\sum_{k=1}^{m}\left|a_{j, k}-\sigma \delta_{j, k}\right|\left|y_{k}\right| \exp \left(i \theta_{j, k}\right), \quad m=1, \cdots, n,
$$

form the vertices of a closed polygon in the complex plane with sides $\alpha_{j, k}=\left|a_{j, k}-\sigma \delta_{j, k}\right|\left|y_{k}\right|$. It is well known that, given any set of $n$ nonnegative numbers $\alpha_{1}, \cdots, \alpha_{n}$, there exists a closed polygon with sides $\alpha_{\kappa}$ if and only if no $\alpha_{k}$ exceeds the sum of the others, $\alpha_{k} \leqq \sum_{j \neq k} \alpha_{j}$ for $k=1, \cdots, n\left[5\right.$, Lemma 5]. Thus $\sigma \in S\left(\Omega_{A}\right)$ if and only if $\exists y \neq 0$, such that $\alpha_{j, k} \leqq \sum_{j \neq k} \alpha_{j, i}$, for $1 \leqq j, k \leqq n$. This is equivalent to (20) with $x_{j}=\left|y_{j}\right| / \sum_{k=1}^{n}\left|y_{k}\right|, j=1, \cdots, n$.

THEOREM 4. $S\left(\Omega_{A}\right)=\bigcap_{\phi} G^{\phi}\left(\Omega_{A}\right)$.

Proof. From the lemma and (19), $\sigma \in S\left(\Omega_{A}\right)$ is equivalent to $\bigcap_{1 \leq, i, j \leqq n} M_{i, j}(\sigma) \neq \varnothing$. By Theorem 2, this is equivalent to $H$ $=\bigcup_{i=1}^{n} M_{i, \phi(i)}(\sigma)$, for each permutation $\phi$ of $1, \cdots, n$, which, in turn is equivalent to $\sigma \in G^{\phi}\left(\Omega_{A}\right)$, for each $\phi$, or $\sigma \in \bigcap_{\phi} G^{\phi}\left(\Omega_{A}\right)$.

If $\sigma \notin S\left(\Omega_{A}\right)$, then there exists a permutation $\psi$ such that $\sigma \notin G^{\psi}\left(\Omega_{A}\right)$ and $H \neq \bigcup_{i=1}^{n} M_{i, \psi(i)}(\sigma)$. By a remark following Theorem $2, \psi$ is the only permutation for which this can occur. Consequently, since the $G^{\phi}\left(\Omega_{A}\right)$ are closed, the boundary of $S\left(\Omega_{A}\right)$ is the union of the boundaries of the sets $G^{\phi}\left(\Omega_{A}\right)$, which implies Corollary 1 of [4].

These results may be extended. If, for $1 \leqq i, j \leqq n$,

$$
\begin{aligned}
& \bar{M}_{i, j}(\sigma)=\left\{x \in H|||\sigma|(-1)^{\delta_{i, j} \delta_{i, j}}+a_{i, j} \mid x_{j}\right. \\
&\left.\leqq \sum_{k \neq j}|| \sigma\left|(-1)^{\delta_{i, j} \delta_{i, k}}+a_{i, k}\right| x_{k}\right\}
\end{aligned}
$$

then condition (12) is satisfied. The generalizations of Theorems 1 and 2 then imply analogues of Theorems 3 and 4, for matrices in the set

$$
\Omega_{A}^{\circ}=\left\{B=\left(b_{i, j}\right)|| b_{i, j}|=| a_{i, j} \mid, 1 \leqq i, j \leqq n\right\} .
$$

This yields the main results of [5]. 
It is also possible to prove the analogue of Theorem 3, for Gerschgorin sets with partitionings [1], [2] using a similar argument. However, in this case, there is no analogue to the lemma characterizing $S\left(\Omega_{A}\right)$ and the extension of Theorem 4 is not generally true. The best results for this case have' been given by Johnston [2].

\section{BIBLIOGRAPHY}

1. D. Feingold and R. S. Varga, Block diagonally dominant matrices and generalizations of the Gerschgorin circle theorem, Pacific J. Math. 12 (1962), 1241-1250.

2. R. L. Johnston, Block generalizations of some Gerschgorin-type theorems, Ph.D. Thesis, Case Institute of Technology, Cleveland, Ohio, 1965.

3. V. Klee, On certain intersection properties of convex sets, Canad. J. Math. 3 (1961), 272-275.

4. B. W. Levinger and R. S. Varga, Minimal Gerschgorin sets. II, Pacific J. Math. 17 (1966), 199-210.

5. - On a problem of O. Taussky, Pacific J. Math 19 (1966), 473-487.

6. R. S. Varga, Minimal Gerschgorin sets, Pacific J. Math. 15 (1965). 719-729.

CASE Institute of Technology 